\title{
RÉFLEXIONS SUR LA SITUATION DU MARGHÉ DE LA CARPE
}

\author{
Par M. le Docteur Emile SCHULMANN \\ Directeur de Magyar Togazdosagok Reszrénytorsag.
}

Il y a un an, nous avons exposé ici, comme suite à un article d'un auteur allemand réputé, comment se présentait, à nos yeux, la crise de la carpiculture (I). Les producteurs sont, maintenant, au seuil d'une nouvelle campagne de vente et les difficultés qui se présentaient déjà l'année passée se sont aggravées dans une mesure inquiétante.

En présence de cette situation, jl semble qu'il y ait lieu d'en dégager les, causes essentielles, celles au moins qui, selon notre opinion, empêchent l'assainissement du marché.

Dans notre précédente communication, nous avions déjà constaté que les éleveurs de Carpes qui se plaignent de la crise perdent souvent de vue que celle-ci ne les éprouve pas seuls; elle s'étend à toutes les branches de l'Agriculture. Si cette généralité du désordre économique se manifestait déjà à l'automne dernier, les circonstances actuelles la mettent encore davantage en relief. G'est pourquoi on ne peut assigner à la gêne de la carpiculture des causes indépendantes de celles de la crise agricole considérée dans son ensemble, dont les principales paraissent bien être :

$I^{\circ}$ La tendance générale de tous les pays européens à restreindre leurs importations ;

$2^{\circ}$ Le fait que les ressources des consommateurs sont devenues trop faibles pour payer des prix assurant aux producteurs une rémunération satisfaisante ;

$3^{\circ}$ La difficulté d'obtenir paiement des fournitures faites à l'étranger.

Les producteurs des pays importateurs de Carpes tâchent d'obtenir une hausse du prix de ce poisson par des mesures qui entravent le commerce international. Ils ne cessent d'exiger de leurs Gouvernements des élévations de droits de douane et souvent même la fermeture de la frontière. La crise générale intérieure leur fournit un argument de poids à l'appui de leur requête.

Mais les Pouvoirs publics ont à tenir compte de la balance du Commerce extérieur; ils ne peuvent donner satisfaction aux vœux des producteurs que dans la mesure où n'est pas compromise l'exportation d'autres marchandises, dont il y a excédent, vers les pays fournisseurs de Carpes. Notam-

(1) Voir Bulletin: - Septembre 1931, p. 70 
ment, les Etats qui vendent à l'étranger plus qu'ils ne lui achètent, - èn premicr lieu l'Allemagne et la Pologne, - ne s'exposeront pas à des représailles de la part de ceux dont ils sont clients pour le Poisson d'étang ; des mesures de rétorsion seraient la conséquence inévitable d'une politique douanièrc exagérément protectionniste.

Celte nécessité de ménager leur clientèle étrangère sera toujours plus importante rue celle d'une protection spéciale des pisciculteurs. C'est pourquoi nous osons dire que les Gouvernements des pays susvisés ne pourront jamais faire aux réclamations de ces derniers un accueil qui les puisse contenter. Il se peut qu'interviennent, à certains moments, en raison de la situation politique actuclle, des mesures prohibitives ; on doit tenir pour certain qu'elles ne seront pas maintenues longrtemps; les intérêts de l'ensemble d'une nation l'emporteront toujours, finalement, sur ceux d'une minorité de citoyens.

Obscrvateur professionnel des mouvements commerciaux de la Carpe sur plusieurs marchés européens, nous nous croyons compétencc pour affirmer que mème un succès des producteurs, faisant prévaloir leurs tendances protectionnistes, ne les conduirait pas au but.

La preuve vient d'en être fournie par la Pologne. Les éleveurs de ce pays ont récemment obtenu une prohibition de l'importation des Carpes durant la saison où la consommation en est la plus forte. En Décembre et Janvier, le poisson de Hongrie, de Russie et de Yougoslavie a été tenu à l'écart du marché. Tout le monde s'attendait à ce qu'une hausse se manifestât, faisant atteindre aux cours le niveau souhaité par les pisciculteurs polonais. Or, il n’en a rien été. Jamais ces cours n'ont été plus bas que durant ces deux mois de fermcture de la frontière. Faute de marchandis étrangère, les consommateurs se sont abstenus, ne voulant pas payer four la Carpe un prix majoré alors que les autres denrées : volaille, viande, etc..., ćtaient en baisse. Bref, les exploitants d'étangs n'ont jamais vendu leur poisson aussi mal qu'au temps de la prohibition d'importation. Pour les motifs indiqués plus haut, le Gouvernement ne put maintenir cette interdiction ; or, elle fut levée sans entraîner une baisse ; au contraire, les prix ont monté d'autant mieux qu'il a été plus facile aux consommateurs d'obtenir les qualités désirées.

Cet exemple frappant dispense d'insister sur l'erreur de la politique nationaliste suivie par les producteurs des pays importateurs de Carpes; cette politique entraine un chaos transitoirc sans atteindre le but escompté par ses promoteurs.

Il est à remarquer que le commerce avec la Pologne pourrait intéresser aussi les éleveurs français, car la distance ne paraît pas prohibitive. En effet, des quantités très considérables de Carpes yougoslaves sont dirigées sur Hambourg où clles arrivent toujours en excellent état; ni le vendeur ni l'acheteur ne se próoccupent de la longueur du trajet.

L'organisation polonaise de la Société d'exploilalion des étangs hongrois (Magyar Togazdosagok Reszénytorsag) a déjà plusieurs fọis examiné 
la possibilité du transport de Carpes françaises à destination de Varsovie, mais divers obstacles, notamment des droits de douanc excessifs, ont, jusqu'ici, empêché les transactions d'aboutir. A l'entrée en Pologne, il faut payer 65 Zlotys par quintal métrique, soit plus encore qu'à la douane allemande, qui perçoit 25 Reichsmarks, taxe déjà exagérée. En outre, les tarifs ayant été établis à une époque où la Carpe se vendait encore à un prix rémunérateur, double de celui actuellement pratiqué, le prélèvement est devenu aujourd'hui relativement fantastique.

La seconde cause fondamentale de la mévente de la Carpe est l'appauvrissement de la masse des acheteurs. Le producteur est forcé de tenir compte de cette circonstance et, pour conserver sa clientèle, d'adapter ses prix au pouvoir d'acquisition du consommateur moyen.

Ici, des remèdes généraux sont nécessaires; les carpiculteurs ne sont guère à même d'intervenir. Il faut attendre, en espérant que les tentatives des grands pays tendant à l'amélioration du Commerce international seront couronnécs de succès. La Conférence économique mondiale actuellement en préparation pourrra contribuer à l'assainissement de la situation, si on réussit à subordonner les intérêts particuliers à l'intérêt général.

Néanmoins, les éleveurs peuvent influer, dans une certaine mesurc, sur la tenue du marché, en s'efforçant d'augmenter le nombre des consommateurs de poisson d'étang. En faisant apprécier ce dernier par des classes de la population qui n'en font point usage, on provoque un accroissement de la demande, par suite une augmentation des prix.

A cet égard, on ne peut guère épargner aux carpiculteurs le reprochè d'avoir, trop souvent, épuisé leur effort à sollíciter de l'Etat des mesures de protection ; faisant peu, parfois même ne faisant rien, pour redresser par eux-mêmes la situation.

On peut, cependant, parvenir à développer la consommation de la Carpe. Il y a de vastes contrées, en Allemagne comme en Pologne, où une population, relativement riche, n'est pas habituée à manger ce Poisson. Voilà le résultat d'un défaut de propagande pour laquelle serait requise la collaboration des importateurs. A cet égard, celle de la Magyar Togazdosagok Resrénytorsag a déjà été offerte ; nous saisissons l'occasion de renouveler cette proposition.

L'an dernier, on a fait, en Hongrie, une active propagande. Des affiches humoristiques ont été apposées pendant des mois dans les rues de Budapest ; le succès a largement dépassé l'attente (Fig. 7). La consommation de la Carpe est en progrès et les restaurants d'été, dans la banlieue de la capitale, l'accommodent en plats exquis dont ils se font réclame.

S'il est regrettable que la population de régions étendues, allemandes ou polonaises, n'achète pas de Carpes, il est surprenant aussi que leur débit, en France, n'ait pas encore pris un développement correspondant à l'importance des étangs et à la situation économique générale du pays. Le fait que poissons de sucre ou de chocolat y sont largement vendus à 
l'occasion du premier Avril, donne à penser qu'on pourrait, à cette époque, populariser la consommation de la Carpe. En Allemagne, la tradition influe considérablement sur la vente. S'il n'y avait la "Sylvestertiarpfen ", - Carpe de Saint-Sylvestre ou, mieux, du jour de l'An, - l'importance du commerce de ce poisson serait fortement réduite. Dans d'autres pays,

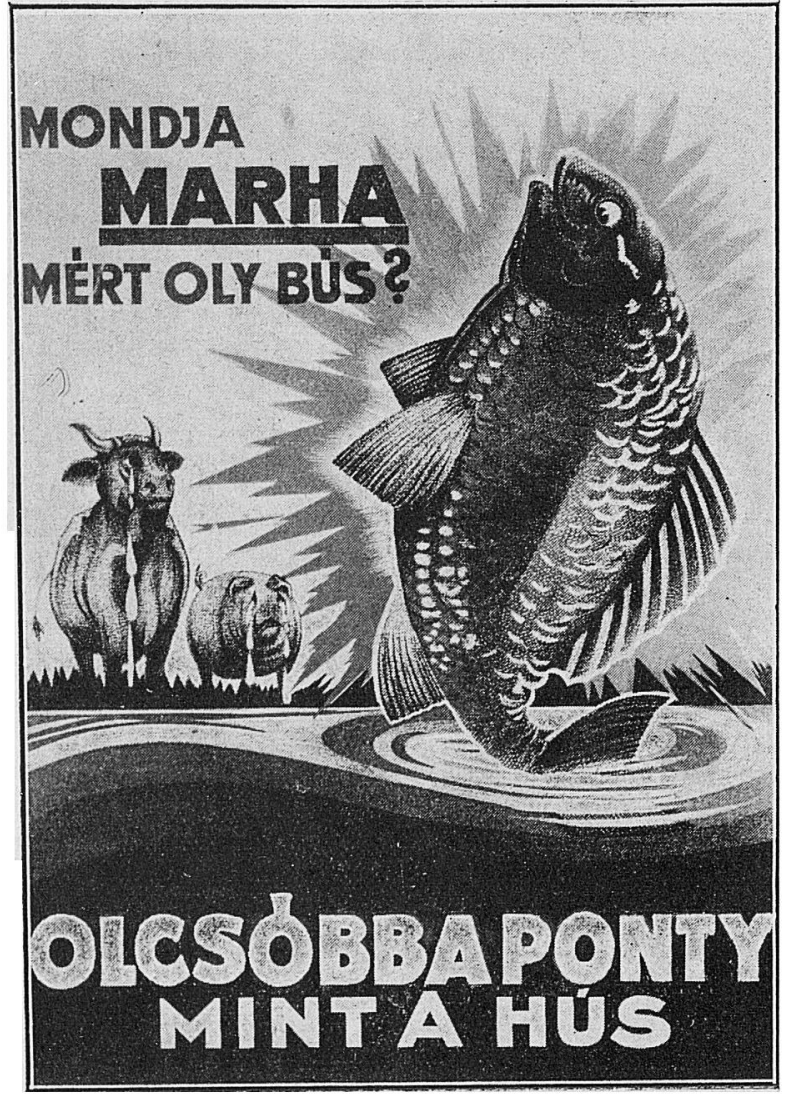

Fig. 7. - Spécimen d'une des affiches éditces pall la Société pour l'exploitalion des etangs hongrois, pour inciter à la consommation de la Carpe.

La légende se traduit comme suit :

- Dis-moi, bour, pourquoi es-tu si triste?

- Parce que la viande est plus chère que le poisson.

on ne peut concevoir un repas de Noël au menu duquel la Carpe ne figurerait pas.

Les observations faites sur d’autres marchés nous donnent à penser, qu'en France aussi, I'ińsuffisance de la consommation tient à ce que le commerce de la Carpe n'cst pas convenablement organisé. Dans aucun pays d'étangs son développement n'a pu marcher de páir avec l'augmentation de Ia production. C'est pourquoi, en Hongrie, comme aussi en Allemagne et en Pologne, les pisciculteurs ont été forcés de s'occuper eux- 
mêmes de la vente de leur poisson et de fonder, à cet effet, des Sociétés coopératives. Ils pourraient, ailleurs aussi, trouver profit à s'engager à leur tour dans cette voie.

Les obstacles au commerce international tenant à des difficultés de paiement ont également un caractère de généralité ; il est souvent compliqué d'obtenir virement en banque de la contrevaleur de marchandises vendues à l'étranger. Mais on ne saurait-en tirer argument pour prohiber l'importation, surtout quand il s'agit de la Carpe, car les transactions dont elle fait l'objet sont dimportance financière trop réduite; elles ne sont pas de celles qui impréssionnent fâcheusement les fonctionnaires des établissements de crédit nationaux contrôlant le paiement des fournitures importées.

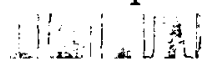

Après avoir passé ainsi en revue les raisons essentielles de la mévente actuelle de la Carpe, il convient de signaler un sérieux motif d'espérer, malgré tout, une orientation plus favorable du commerce pour la campagne à venir.

Tandis qu'au cours des dernières années, le rendement des étangs a toujours été croissant, il est presque certain que pour la prochaine saison des pêches, une diminution assez forte est à prévoir. Sauf la France, tous les pays producteurs sont très dépourvus de capitaux. Les agriculteurs surtout souffrent de cette pénurie ; leurs dettes sont allées grossissant sans cesse, tandis que le bilan de leurs exploitations devenait de plus en plus défavorable. Spécialement, en carpiculture, le prix du nourrissage a augmenté alors que le prix de la Carpe s'abaissait fortement. Il s'en est suivi une réduction de la production et, si la circulation normale n'était pas troublée par des mesures protectionnistes, la diminution de l'offre devrait provoquer une hausse.

La question qui se pose actuellement est de savoir si ce résultat sera obtenu avec les entraves à la liberté du commerce, entraves qui ne peuvent pas être durables et, surtout, sont inopérantes en tant que remède à la crise. Pour autant qu'on puisse actuellement formuler un pronostic, les perspectives seraient plutôt favorables. Nous avons, pour notre part, la conviction que les nécessités commerciales l'emporteront, que les mesures inconsidérées de défense douanière seront reconnues vaines. Aussi conclurons-nous en exprimant le vœu que les carpiculteurs conservent leur sangfroid et ne se laissent pas inciter, par crainte du pire, à une vente défavorable de leur prochaine récolte. Au lieu de se résigner passivement, ils doivent tout faire pour s'opposer aux prétentions d'un nationalisme outrancier et pour développer, par une propagande active, la consommation du poisson d'étang. 